\title{
Measurement of Sound Pressure Levels in Anechoic Chamber and a Noisy Environment Experimentally
}

\author{
Mohammad Al Zubi \\ Mechanical Engineering Department, Tafila Technical University, Tafila, Jordan \\ Email: mohmmad1972@yahoo.com
}

How to cite this paper: Al Zubi, M. (2018) Measurement of Sound Pressure Levels in Anechoic Chamber and a Noisy Environment Experimentally. Open Journal of Acoustics, 8, 13-22. https://doi.org/10.4236/oja.2018.82002

Received: March 1, 2018

Accepted: April 1, 2018

Published: April 30, 2018

Copyright $\odot 2018$ by author and Scientific Research Publishing Inc. This work is licensed under the Creative Commons Attribution International License (CC BY 4.0).

http://creativecommons.org/licenses/by/4.0/

\section{(c) (i) Open Access}

\begin{abstract}
In real life, when a noise problem occurs, it is important to identify the cause and measure the noise of the source, since it may affect human beings or other constructions due to vibration generated from noise, so it is necessary to determine the noise related to a specific source like a machine in the presence of other sources which is a very important approach in noise control engineering. In this article a full experiment was executed to measure the sound pressure levels of various sources (stationary and non-stationary), in both an anechoic chamber and a non-ideal noisy environment. The sound pressure level was extracted for different sources and compared for both ideal and non-ideal environment. The results showed that acoustical free field of the space is the best field to do measurements to avoid reflection, on the other hand the difference between the source and the background should be more than $3 \mathrm{~dB}$ to get better results.
\end{abstract}

\section{Keywords}

Sound Pressure Level, Anechoic Chamber, Free Field, Noisy Environment, Sound Extraction

\section{Introduction}

Acoustics is the science of sound [1], it is considered one of the most important disciplines in mechanical engineering since it treats with many engineering problems related to daily life like traffic noise, wind farms noise, industrial zones noise, and many other sources, so industrial noise control becomes a branch of interest for many researchers since it is related directly to human health [2]

Sound field may be excited by a number of sources, at a given point, the 
acoustic pressure $p(t)$, is the sum of all waveforms $p_{s}(t),(s=1,2,3, \ldots)[3]$, caused by the individual sources. When only one source is of interest, all other sources are considered as background noise [4]. Free field means propagation of sound in space without reflections [5]. This condition can be achieved in anechoic chamber in which all the walls including the floor and the ceiling are covered with a highly absorptive material to avoid reflection [6]. Figure 1 shows a wall in the anechoic chamber in which the design was made according to ISO 3745 [7], to ensure free field conditions inside the chamber [8]. This paper provides an overview of the main experimental techniques employed for measuring sound pressure levels in ideal and non-ideal environments which was not done before.

\section{Sound Pressure Level and Decibels}

Sound pressure level (SPL) uses the decibel logarithmic unit of sound pressure (dB) named after Alexander Graham Bell, to express the wide range of sound pressures perceptible to humans in two or three digits [9] [10] [11]:

$$
p_{\text {rms }}=\left(p_{\text {ref }}\right) * 10^{\mathrm{sp} / 20}
$$

SPL in decibels is 20 times $\left(\log _{10}\right)$ of the rms (square root of the mean square pressure acoustic pressure) relative to a reference pressure, The commonly used "zero" reference acoustic pressure in air is $20 \mu \mathrm{Pa} \mathrm{rms,} \mathrm{which} \mathrm{is} \mathrm{usually} \mathrm{consi-}$ dered the threshold of human hearing (at $1 \mathrm{kHz}$ ) which is a pressure of $0 \mathrm{~dB}$. Figure 2 shows how to measure SPL [9].

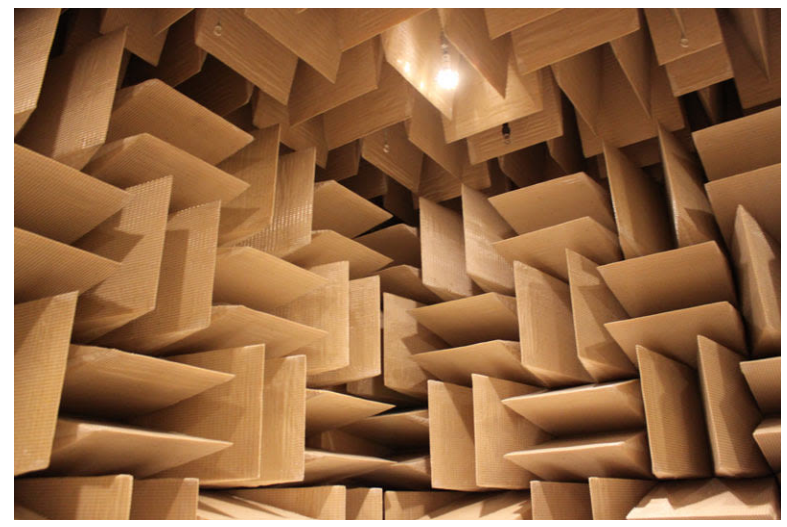

Figure 1. Side of the anechoic chamber in which the experiment took place.

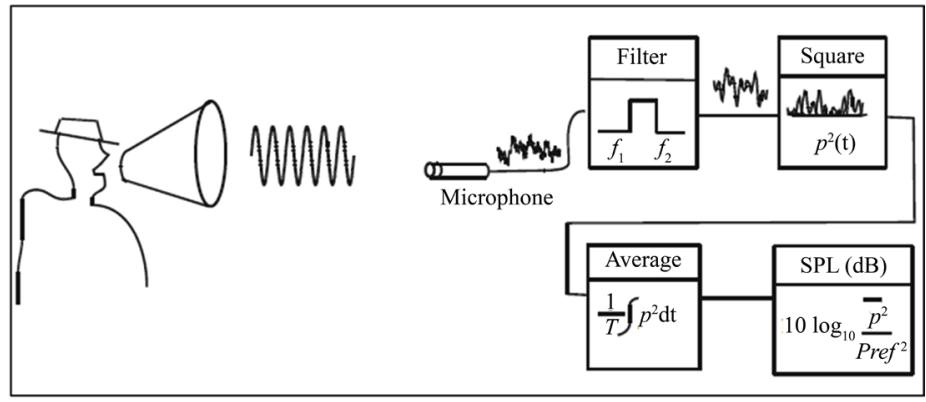

Figure 2. Measuring of Sound Pressure Levels [9]. 
SPL can be calculated due to a given source $\left(L_{s}\right)$ from measurements taken in the presence of background noise $\left(L_{b g}\right)$, in which $L_{c o m b}$ is the combined SPL from source and background, is given by the following equation [12]:

$$
\begin{aligned}
& 10^{L_{s} / 10}=10^{L_{\text {comb }} / 10}-10^{L_{b g} / 10}, \text { or } \\
& L_{s}=10 \log \left(10^{L_{\text {comb }} / 10}-10^{L_{b g} / 10}\right)
\end{aligned}
$$

The sound pressure level can be calculated using the following equation:

$$
L p=20 \log (P / \operatorname{Pr}) \text { in } \mathrm{dB} \text {, and, } \operatorname{Pr}=20 \text { micro-Pascals }
$$

where: $L_{s}$ : The source sound pressure level in $\mathrm{dB}$. $L_{\text {comb }}$ : The combined source sound pressure level in $\mathrm{dB} . L_{b g}$ : The background sound pressure level in $\mathrm{dB}$.

The main objective from this experiment is to measure the sound pressure levels of various sources (stationary and non-stationary), in an anechoic chamber and in a noisy environment, then to extract sound pressure level for different sources and compare the results between ideal and actual environments. Stationary source as shown in Figure 3 means "Wave patterns created by a stationary sound source $S$ are symmetric in every direction" [13], in which the sound velocity ( $c_{s}$, and the wave length $\lambda$ are shown), while non stationary sound signal means that spectral content changes with time [14]. Background noise or ambient noise is defined as any sound other than the sound being monitored.

\section{Experimental Setup}

The major components used to execute the experiment were:

1) Sound source generated from a laptop them emitted through a loud speaker.

2) Anechoic chamber (ideal condition), and a room (which represents the real environment) to carry out the measurements in both ideal and non-ideal environments.

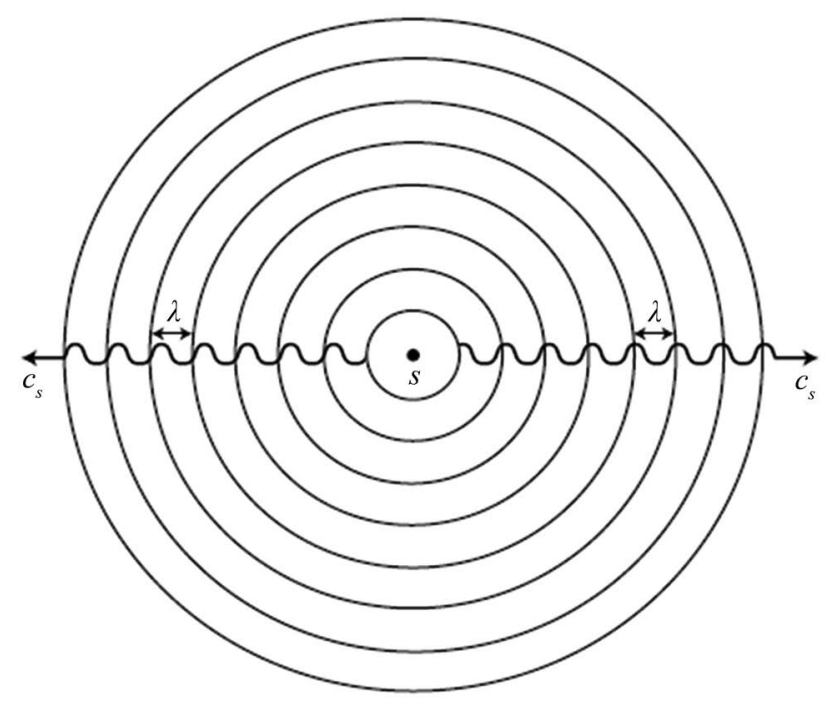

Figure 3. Stationary sound source [13]. 
3) Bruel and Kjear (B \& K) microphone, to measure the sound pressure level from different sources, the microphone was connected to two channel analyzer and the signal was sent to a laptop in which the software is installed to execute the calculations and get the results.

4) Different connections to connect the hardware parts.

5) A calibrator to calibrate the microphones before starting the measurements, according to standards, this calibrator is a piston phone B \& K type 4220, Serial No.704798 [15].

6) National instrument 2-channel data acquisition system type NI 9234.

Different photos were taken to illustrate the experiential setup, Figure 4 shows the sound source, the microphone, both inside the anechoic chamber, the distance from source to microphone was (24 inches), finally the laptop and the data acquisition system used in the experiment.

Figure 5 shows the arrangement inside the real room, in which two sources were used, one as a background, and the other is the source, the distance between speakers and microphone was 24 inches.

The frequency band used in this experiment was a (1/3) octave band. To clarify the concept of octave band, it should be mentioned here that linear frequency scale $(100 \mathrm{~Hz}, 200 \mathrm{~Hz}, 300 \mathrm{~Hz}$, etc.) is inconvenient for assessing acoustical frequencies [13], which range from $20 \mathrm{~Hz}$ to $20,000 \mathrm{~Hz}$, it is more convenient to separate this audible frequency range into ten unequal segments called

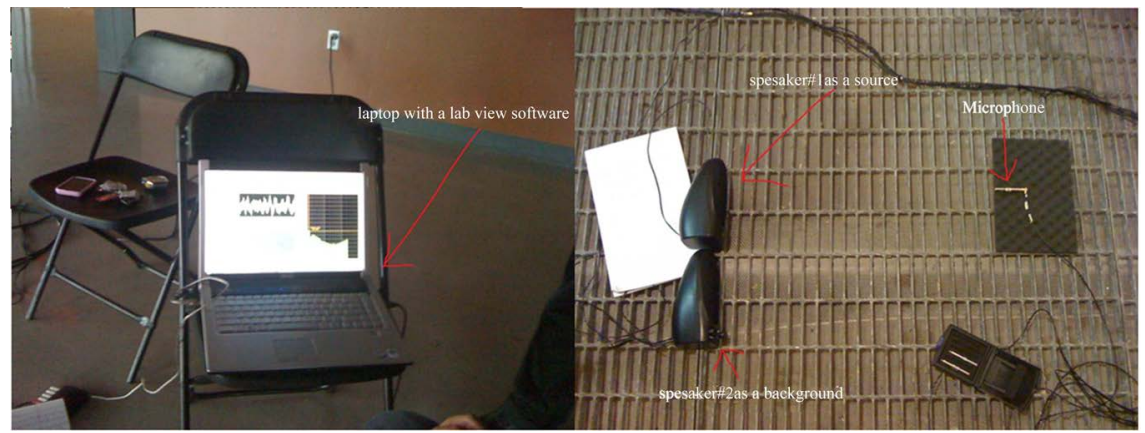

Figure 4. Test setup including (the speakers, the microphone, and the software) in the anechoic chamber.

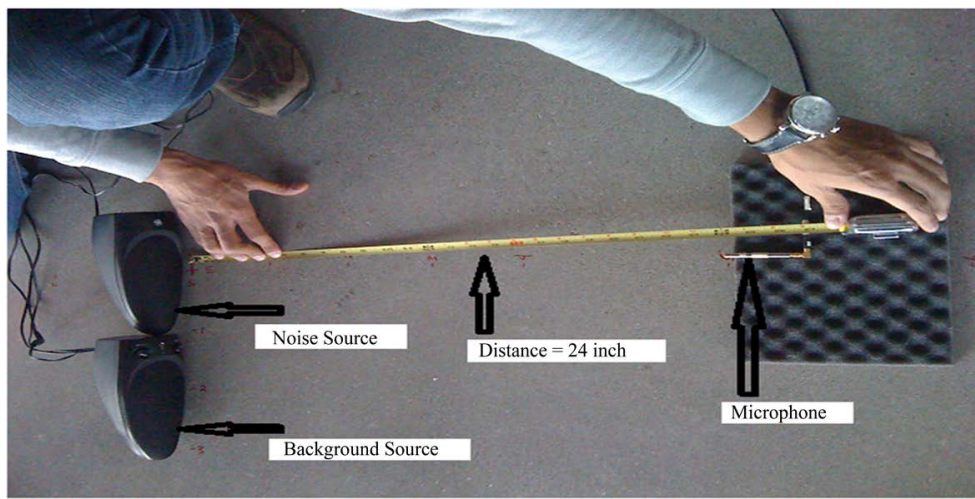

Figure 5. Measuring the distance between source and microphone (24 inches). 
octave bands, an octave higher means a doubling in frequency; e.g., $63 \mathrm{~Hz}, 125$ $\mathrm{Hz}, 250 \mathrm{~Hz}$, etc. [13]. On the other hand, one third octave band means that the octave band is separated into three ranges, this band is mainly used in environmental and noise control applications, since it provides a further in-depth outlook on noise levels across the frequency composition [16]. The averaging time was 30 seconds, and this is simply the time for calculating the average of data. The laptop in which the software is installed was connected to the data acquisition system, then the data acquisition system was connected to both the speaker and the microphone, the signal was generated from the laptop and the output noise was emitted through the speaker, the signal was captured by the microphone and then resent to the laptop through the data acquisition system, the signal was analyzed by the software and the SPL for each frequency can be read, the different values for different sources were utilized to extract any source noise of interest. The first step of the experiment was to measure the SPL of the ambient environment and different sources inside the anechoic chamber $\left(f_{\text {cut-off }}=\right.$ $120 \mathrm{~Hz}$ ), those measurements were considered as a bench mark measurements, the sound sources used in the experiment were (white noise, synthesized noise, harp music, NPR news),in which the first two sources are stationary and the other two are non-stationary, to avoid reflection of sound the speaker was laid on the floor inside the anechoic chamber, then the microphone was laid on a pad at a distance of 24 inches from the source, this distance is the distance in which free field was detected inside the actual room space [5].

\section{Results and Discussion}

The experiment was started by measuring the ambient in the anechoic chamber, then, four different sounds (Harp music, White noise, NPR news and Synthesized sound) were measured in the anechoic chamber, the next step was measuring mixed noises (Harp music and synthesized), (Harp music and white noise) in the anechoic chamber. Moreover, synthesized sound and white noise were measured as a background in the anechoic chamber. Same measurements were repeated in the room.

The excel files exported from the software have two readings, the frequency, and the SPL for the measured sound. The charts showed the SPL versus distance for each direction and for the frequency band from $(100 \mathrm{~Hz}-20 \mathrm{kHz})$.

The first comparison was taken between ambient measurements in both rooms as shown in Figure 6.

It can be seen that the data converge as the frequency increases; this may be due to the fact that after the cut off frequency of the room $(2000 \mathrm{~Hz})$, the data becomes more accurate.

The other comparison was made between Harp music inside anechoic chamber; this was done by extracting harp music from:

1) Combined (Harp \& ambient), background (ambient).

2) Combined (Harp \& white,), background (white) 


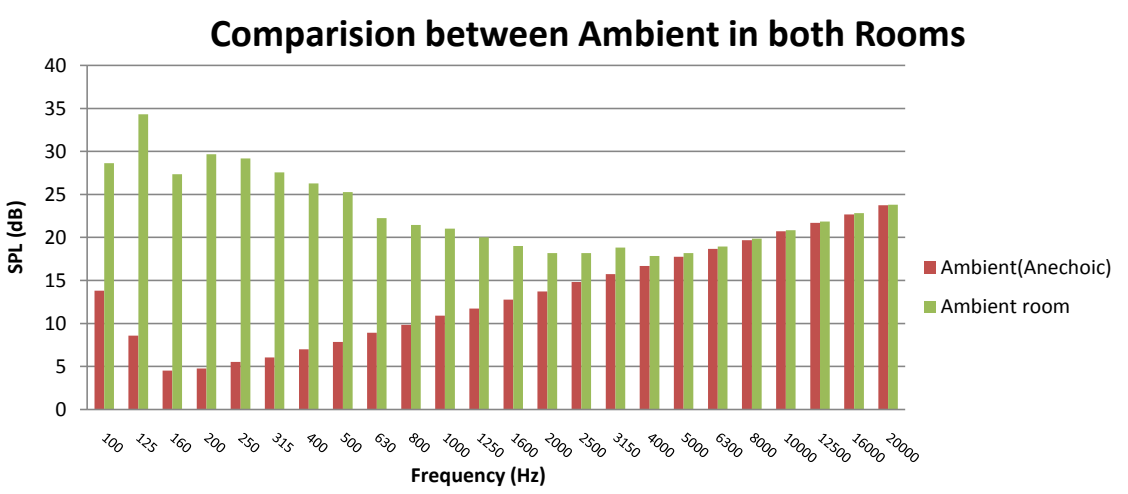

Figure 6. SPL as function of frequency (Anechoic and room).

3) Combined (harp \& synthesized), background (synthesized)

As it can be seen from the Figure 7, the results were good in the frequency range $(160-1600 \mathrm{~Hz})$, however below or above this range, there are some results that can be ignored, because the background or ambient SPL exceeds the combined noise SPL, in such a case the equation can't be used. The source types either stationary or non-stationary didn't affect the extracted results.

Figure 8 shows the extracted Harp from different combinations in the real environment, from the graph, the results were good in the frequency range (250 - $630 \mathrm{~Hz}$ ), at different frequencies either below or above this range; some results can't be used because the background exceeds the combined SPL value.

H-A: Pure harp extracted from Harp \& ambient.

$(\mathrm{H}+\mathrm{S})-\mathrm{S}$ : Harp extracted from combined Harp \& Synthesized (Synthesized as background).

$(\mathrm{H}+\mathrm{W})-\mathrm{W}$ : Harp extracted from combined Harp \& White (White as background).

(H+NPR)-NPR: Harp extracted from combined Harp \& NPR (NPR as background).

It should be noted here that word synthesize means to produce by combining separate elements. Thus, synthesized sound is sound that a musician builds from component elements, while sound synthesis means the electronic production of sound where no acoustic source is used [17] [18].

Figure 9 shows the synthesized noise in room extracted from combined synthesized and ambient, with ambient was used as background, the other case is synthesized extracted from combined synthesized and white noise, with white noise as a background.

Again from Figure 9, some results are good, others are not, the anechoic chamber results can't be calculated because the background SPL exceeded the source SPL, this is may be due to the fact that background noise was louder than the source noise.

Figure 10 shows the white noise in the room extracted from combined white and ambient, with the ambient as background, the other case was white extracted from combined synthesized and white, with synthesized as a background, 


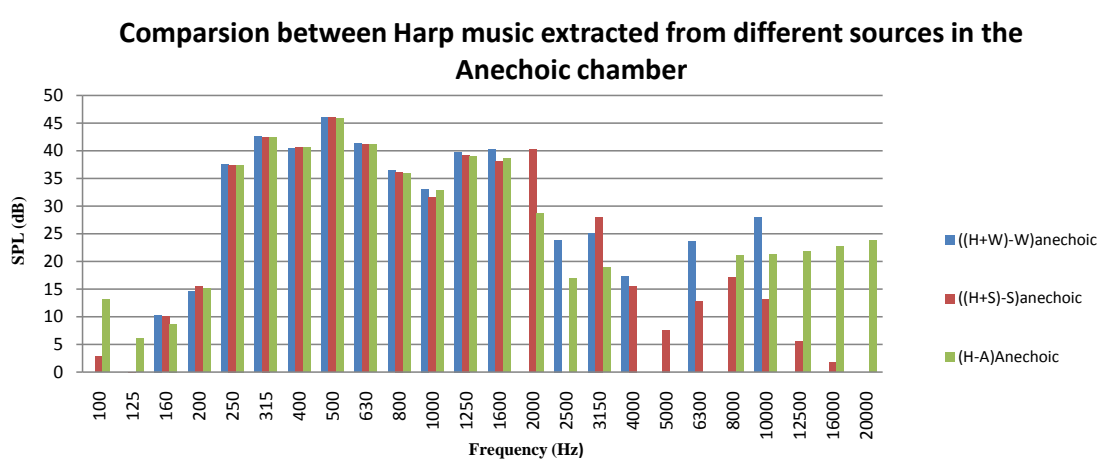

Figure 7. Comparison between extracted Harp in Anechoic chamber.

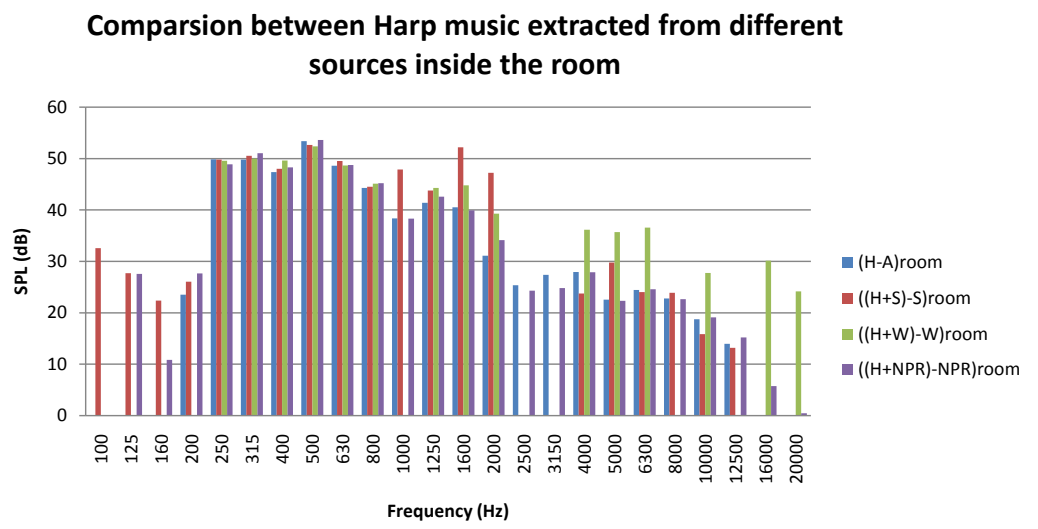

Figure 8. Extracted harp from different combinations in real environment.

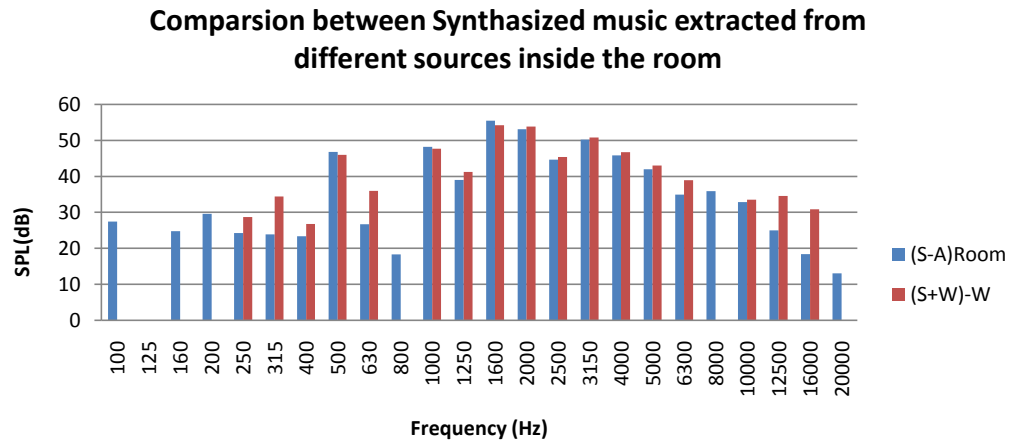

Figure 9. Extracted synthesized from different combinations in real environment.

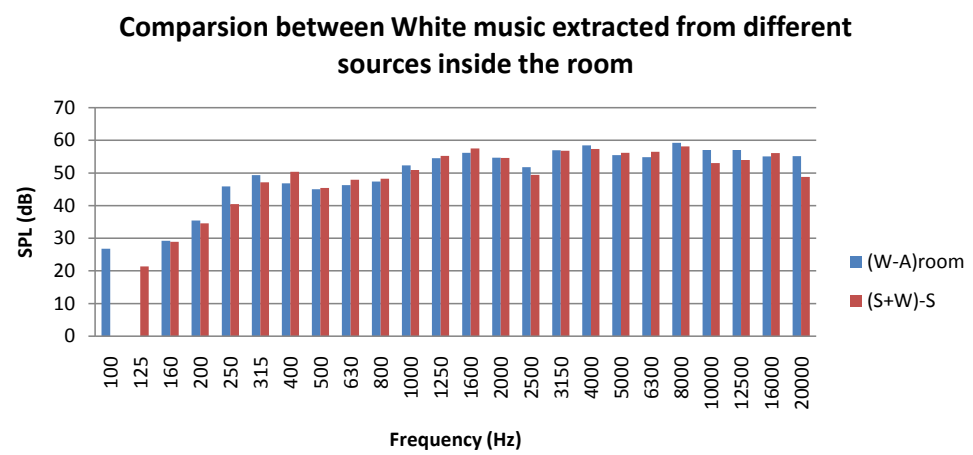

Figure 10. Extracted white noise from different combinations in real environment. 
comparing Figure 9 and Figure 10, shows that the results here were more reasonable, this is due to the fact that in the majority of measurements the sound SPL exceeds the background SPL by more than $3 \mathrm{~dB}$. It should be noted that the following abbrivations are related to Figure 10:

1) (W-A): Combined white and ambient (W), (A) is ambient as background

2) $(\mathrm{S}+\mathrm{W})-\mathrm{S}$ : Combined synthesized and white noise, with synthesized as background

From Figures 11-14, a comparison was made between the same sources in the two environments. For all cases it is obvious that there is a difference between the two environments, in real environment the SPL is higher, this is due to the reflections in the room, and the noise emitted from other sources like air conditioning unit, cars in the street, etc. It can be observed that the source type either stationary or non-stationary doesn't affect the results. Again some measurements disappear from the figures due to the fact that background sound exceeds source sound.

Figure 15 is a Harp extracted from different combinations in both environments, in this figure the results related to both environments (anechoic and real room) coincide, which means the measurements were correct, however, there is a difference in the results for the same frequency in both environments, it is higher in the real room, again some results in each environments can't be calculated as background noise exceeds source noise.

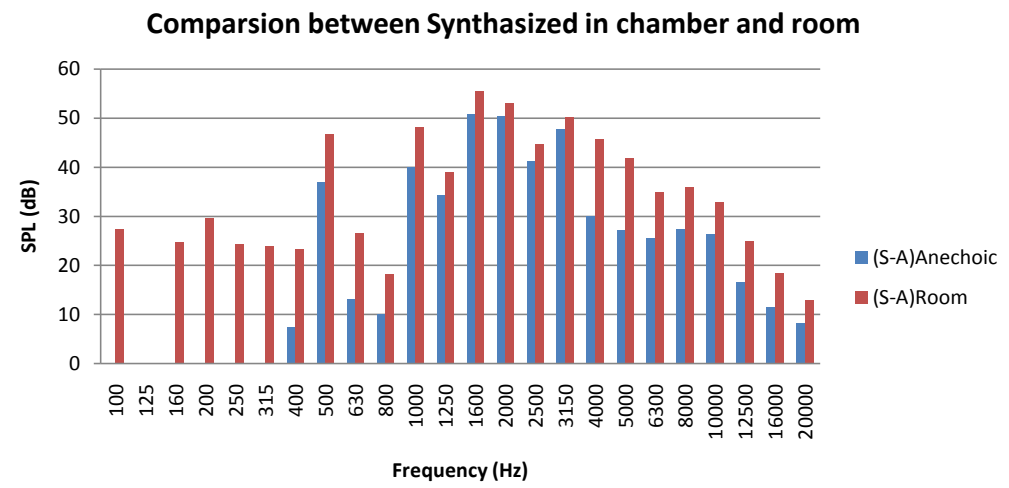

Figure 11. Synthesized in both environments, where (S-A) Pure synthesized extracted from synthesized \& ambient.

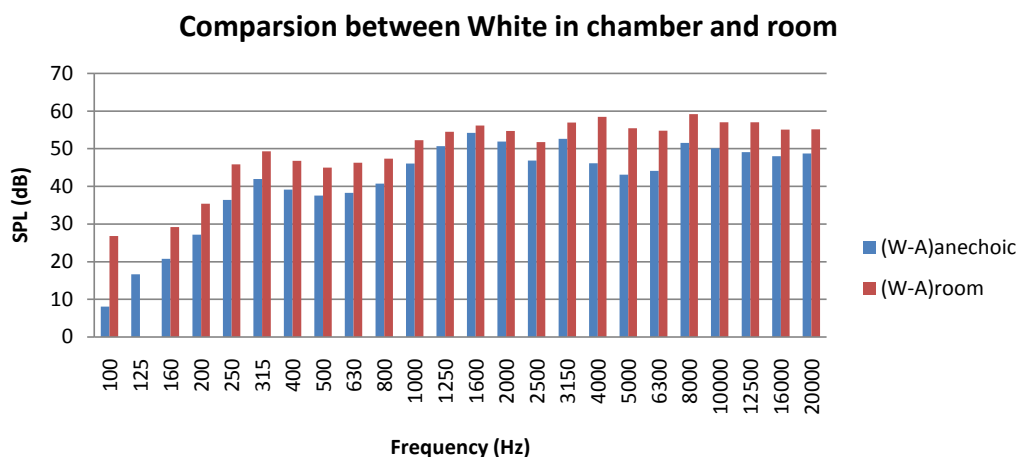

Figure 12. White in both environments. 


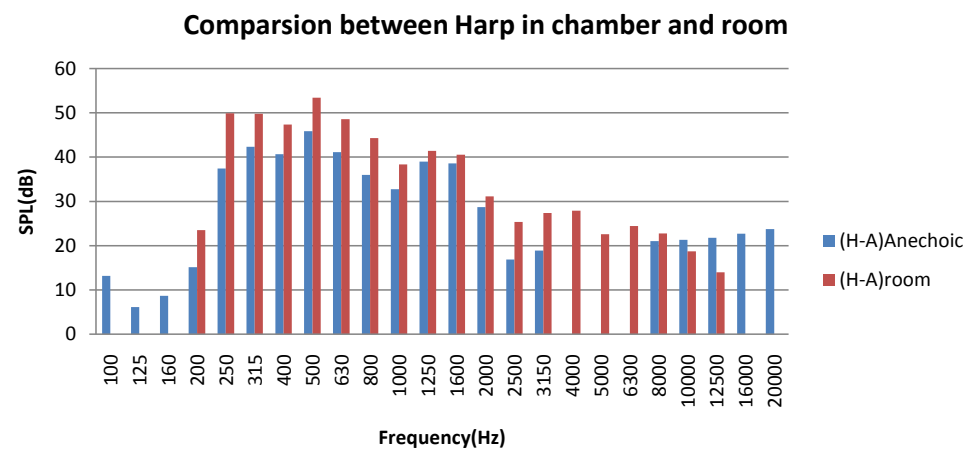

Figure 13. Harp in both environments.

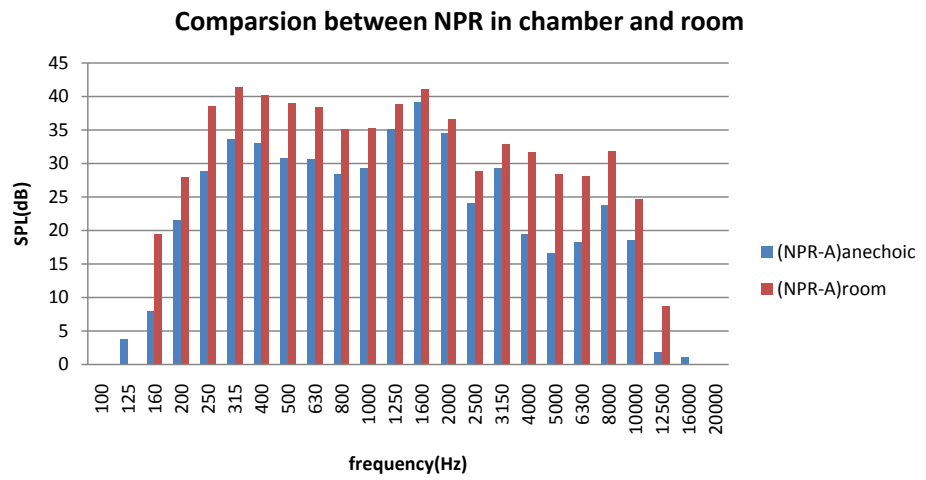

Figure 14. NPR in both environments.

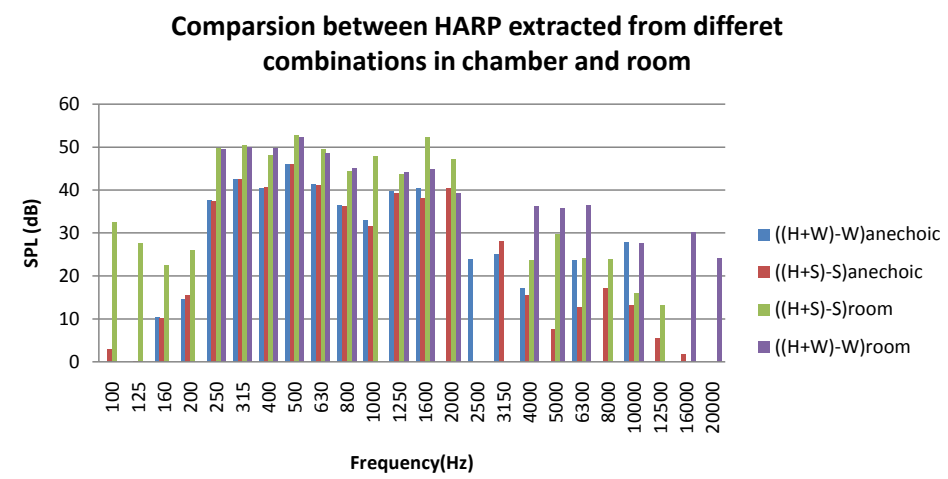

Figure 15. Comparison between Harp SPL extracted from different sources in both environments.

\section{Conclusions}

This article deals with an important issue in noise control problems, and it gives a scientific point of view on how to deal with problems in real life. It was shown how to extract any noise source from a combination of sources if the background is known. An important conclusion is that if the source type is either stationary or non-stationary, it will not affect the results. The difference between the source and the background should be more than $3 \mathrm{~dB}$ to be in the free field zone to get better results and avoid reflection. Finally the background noise shouldn't be louder than the source to guarantee precise results. 


\section{References}

[1] Anselmet, F. and Pierre-Olivier, M. (2016) Acoustics, Aeroacoustics and Vibrations. Wiley-ISTE.

[2] Harris, M.K. (2002) Welding Health and Safety: A Field Guide for OEHS Professionals. American Industrial Hygiene Association, Falls Church, VA.

[3] Munjal, M.L. (2013) Noise and Vibration Control, IISc Lecture Notes Series. Indian Institute of Science, India. World Scientific Publishing Co. Pte. Ltd.

[4] Dahl, P.H., Miller, J.H., Cato, D.H. and Andrew, R.K. (2007) Underwater Ambient Noise. Acoustics Today, 3, Issue 1.

[5] Al Zubi, M. (2018) An Experimental Method to Determine the Cut-Off Frequency of an Acoustical Free Field in a Non-Ideal Environment. Open Journal of Acoustics, 8, 1-11. https://doi.org/10.4236/oja.2018.81001

[6] (1989) Acoustic Noise Measurements. 5th Edition, Bruel\& Kjaer, Denmark. https://www.bksv.com/media/doc/br0047.pdf

[7] (2003) ISO, ISO 3745, Acoustics-Determination of Sound Power Levels of Noise Sources-Precision Methods for Anechoic and Semi-Anechoic Rooms. International Organization for Standardization, Geneva.

[8] Kopiev, V.F., Palchikovskiy, V., Bersenev, Yu.V., Makashov, S.Yu., Belyaev, I.V., Korin, I.A., Sorokin, E.V., Khramtsov, I.V. and Kustov, O.Yu. (2017) Design and Qualification of an Anechoic Facility in PNRPU. Procedia Engineering, 176, 264-272.

[9] Blevins, R.D. (2016) Formulas for Dynamics, Acoustics and Vibration. Wiley, Hoboken.

[10] Thomson, W.T. (1988) Vibration Theory and Applications. Prentice Hall, Upper Saddle River.

[11] Leissa, A.W. (2005) Historical Bases of Rayleigh and Ritz Methods. Journal of Sound and Vibration, 287, 961-978. https://doi.org/10.1016/j.jsv.2004.12.021

[12] Berge, E.H. (2003) The Noise Manual Revised. 5th Edition, American Industrial Hygiene Association, Falls Church, VA.

[13] Hirose, A. and Lonngren, K.E. (2010) Fundamentals of Wave Phenomena. 2nd Edition, SciTech Publishing, New York.

[14] Priemer, R. (2013) Matlab for Electrical and Computer Engineering Students and Professionals with Simulink. SciTech Publishing, New York.

[15] https://www.bksv.com/en/products/transducers/acoustic/calibrators/4228

[16] Crocker, M. (2007) Handbook of Noise and Vibration Control. Wiley, Hoboken.

[17] Truax, B. (1999) Handbook for Acoustic Ecology. 2nd Edition, ARC Publications, Todmorden.

[18] Berger, E.H., et al. (2003) The Noise Manual (Revised). 5th Edition, American Industrial Hygiene Association, Falls Church, VA. 